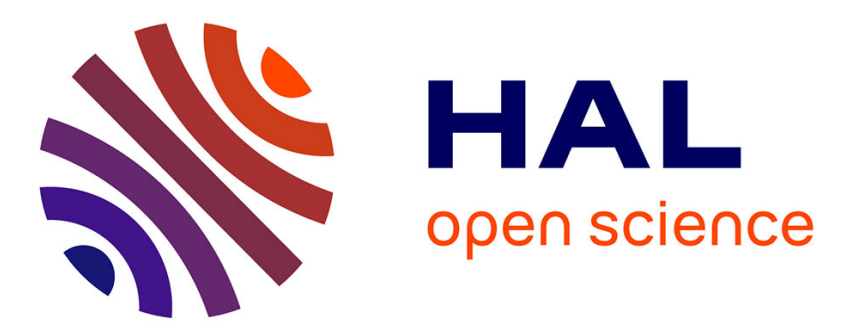

\title{
Le rôle de l'organisation du travail des capital investisseurs dans la construction de relations interpersonnelles avec les entrepreneurs
} Claire Champenois, Michel Devigne, François-Régis Puyou

\section{- To cite this version:}

Claire Champenois, Michel Devigne, François-Régis Puyou. Le rôle de l'organisation du travail des capital investisseurs dans la construction de relations interpersonnelles avec les entrepreneurs. Finance Contrôle Stratégie, 2017. hal-01709993

HAL Id: hal-01709993

https://hal-audencia.archives-ouvertes.fr/hal-01709993

Submitted on 15 Feb 2018

HAL is a multi-disciplinary open access archive for the deposit and dissemination of scientific research documents, whether they are published or not. The documents may come from teaching and research institutions in France or abroad, or from public or private research centers.
L'archive ouverte pluridisciplinaire HAL, est destinée au dépôt et à la diffusion de documents scientifiques de niveau recherche, publiés ou non, émanant des établissements d'enseignement et de recherche français ou étrangers, des laboratoires publics ou privés. 


\title{
Le rôle de l'organisation du travail des capital investisseurs dans la construction de relations interpersonnelles avec les entrepreneurs
}

\author{
Claire CHAMPENOIS \\ Audencia Nantes-Ecole de Management \\ Michel DEVIGNE \\ Ecole des Mines de Nantes-LEMNA \\ François-Régis PUYOU \\ Audencia Nantes-Ecole de Management
}

Classification JEL: G24 - Venture capital ; L26 - Entrepreneurship ; G34 - Buyout

Réception :; Acceptation:

Correspondance : cchampenois@audencia.com

Résumé : L'article, fondé sur une étude de cas d'une société de capital investissement, complète les travaux qui ont souligné l'importance des liens interpersonnels entre entrepreneurs et investisseurs en précisant l'importance des règles organisationnelles dans la création et le maintien de ces relations. Il montre comment l'organisation du travail des capital investisseurs (i.e. des règles formelles et informelles, des procédures et des dispositifs encadrant le travail) permet à ces derniers de construire des liens étroits avec les entrepreneurs tout au long des étapes de l'activité d'investissement. Ce faisant, l'article étend l'analyse de ces relations aux phases de pré-investissement et introduit une dimension organisationnelle à la littérature sur la valeur ajoutée non financière, en précisant les effets bénéfiques cruciaux de mécanismes de type bureaucratiques sur le développement de relations très personnalisées.

Mots-clés: Capital investissement, valeur ajoutée non financière, entrepreneurs, dirigeants.

Abstract: The article enriches the literature on non-financial valueadded, which underlines the importance of interpersonal relationships 
between entrepreneurs and investors. It draws on a case study of a private equity firm. It shows how the organization of the investors' work (i.e. formal and informal rules, procedures and systems framing this work) enable investors to develop close relationships with entrepreneurs during all stages of the investment activity. The article introduces an organizational dimension that is often overlooked and broadens the analysis to include pre-investment stages.

Key words : Private equity, non-financial value-added, entrepreneurs, CEOs.

Les capital investisseurs apportent des financements aux entrepreneurs sous forme de participations en capital. Le fait de s'engager auprès d'entreprises généralement non cotées pour lesquelles la disponibilité des informations est faible (Desbrières 2001) les incite fortement à établir un contrôle étroit de celles-ci (e.g. Sapienza et al. 1996; Busenitz et al. 2004). L'apport de capital et le contrôle ne sont cependant pas les seuls rôles remplis par les capital investisseurs : ces derniers apportent aussi de la «valeur ajoutée » aux entreprises en faisant valoir leurs expériences et connaissances auprès des dirigeants (e.g. Bygrave et Timmons 1992 ; De Clercq et Sapienza 2001 ; Large et Muegge 2008). Ils s'assurent aussi de la bonne coopération des entrepreneurs identifiés comme des acteurs essentiels du succès de l'entreprise et jamais seulement comme des agents opportunistes à surveiller. Les entrepreneurs sont souvent en effet détenteurs d'une expertise unique et parfois d'une part importante du capital, leur conférant le statut de partenaires incontournables et puissants (Arthurs et Busenitz 2003).

Au-delà de l'apport de capital et de la relation de contrôle, la littérature sur le capital investissement a commencé «à ouvrir la 'boite noire' de la relation capital risqueur-entrepreneur $\gg$ (De Clercq et Manigart 2007, p. 194, traduit par nous) en mettant l'accent sur les apports non financiers des premiers aux seconds (Gorman et Sahlman 1989 ; Hellmann et Puri 2002). Notamment le courant de recherche dédié à l'étude de la valeur ajoutée non financière apportée par les capital investisseurs (Sapienza, 1992 ; Busenitz et al. 2004 ; Politis 2008) enrichit grandement la compréhension de la relation entre les deux parties. Il met en lumière les processus de transfert de cette valeur ajoutée non financière au moyen d'une relation personnalisée, c'est-à- 
dire liée aux personnes de l'investisseur et l'entrepreneur en présence et aux sentiments «intuitu personae», mélanges de proximité et de complémentarité. Ces travaux soulignent que la «qualité » des relations interpersonnelles (De Clercq et Sapienza 2001, 2006) entre les deux partenaires est un élément essentiel pour la performance des entreprises soutenues. Pourtant, cette relation est généralement étudiée en dehors $\mathrm{du}$ contexte organisationnel dans lequel les capital-investisseurs travaillent. Les recherches laissent ouverte la question à laquelle tente de répondre cet article: comment s'articulent la dimension personnalisée de la relation entrepreneur-investisseur et les règles génériques, applicables sans distinction de personnes, que produisent les sociétés de capital-investissement ? Nous montrerons ainsi le rôle joué par les règles organisationnelles dans la construction de relations personnalisées, c'est-à-dire adaptées aux attentes et aux personnalités des entrepreneurs avec lesquels les capital investisseurs ne cessent d'être en contact. Cette question est cruciale afin de mieux comprendre les pratiques managériales susceptibles au sein des sociétés de capital investissement d'encourager l'établissement de relations interpersonnelles de qualité avec les entrepreneurs.

Les résultats présentés reposent sur l'analyse d'un cas de société de capital investissement où le développement de telles relations par les capital investisseurs fait l'objet d'un accompagnement systématique par le biais de normes et de mécanismes organisationnels mis en place par l'entreprise qui les emploie. L'accent est mis sur le travail réalisé par la société d'investissement considéré, d'un point de vue conceptuel, comme une «action collective organisée » (Friedberg 1993). Autrement dit, sont étudiées les pratiques de travail des acteurs telles qu'elles ressortent comme encadrées et modelées par des règles formelles et informelles, des procédures, des dispositifs, des normes stabilisés et partagés par les membres de l'organisation. L'étude couvre les cinq étapes classiques de l'activité d'investissement définies par Tyebjee et Bruno (1984) (identification de l'affaire, sélection, évaluation, structuration, activités post investissement) et même audelà, avec une logique anticipative des deals futurs.

L'article commence par une revue de littérature montrant que les travaux sur la valeur ajoutée non financière ont souligné la dimension personnalisée des relations entre entrepreneurs et capital investisseurs (1.). Les théories de la bureaucratie offrent un socle théorique permettant de penser les règles produites par les organisations que sont les sociétés d'investissement (2.). La méthodologie de recherche est 
ensuite présentée (3.). Les mécanismes de l'organisation du travail des directeurs de participations leur permettant de construire et préserver des relations interpersonnelles avec les entrepreneurs ${ }^{1}$ à chaque étape de la vie de la participation sont alors analysés (4.). Une dernière section discute les résultats présentés et conclut (5.).

\section{Des relations personnalisées de type "intuitu personae » comme modalité cruciale de transmission de la valeur ajoutée non financière}

Les travaux de Rosenstein (1988; Rosenstein et al., 1989, 1993 ; Macmillan et al. 1989 ; Gorman et Sahlman 1989 ; Sapienza 1992) ont été les premiers à mettre l'accent sur la diversité des apports non financiers des capital investisseurs. Les multiples dimensions de la valeur ajoutée non financière apportée par les capital investisseurs en phase de post-investissement (De Clercq et Manigart 2007) ont depuis été largement documentées. La recension de ces recherches par Large et Muegge (2008) permet de catégoriser ces apports non financiers en huit types : deux concernent la valeur ajoutée orientée vers l'environnement de l'entreprise (Legitimation, Outreach) et six la valeur ajoutée orientée vers l'intérieur de l'entreprise (Recruiting Activities, Mandating, Strategizing, Mentoring, Consulting et Operating). En revanche, la question de savoir comment cette valeur ajoutée est effectivement transférée aux entrepreneurs a été relativement négligée. Ce n'est que dans un second temps que des recherches ont commencé à distinguer ce qui relève du «contenu» de la valeur ajoutée apportée par les investisseurs, des «processus » qui permettent le transfert de cette valeur ajoutée (De Clercq et Manigart 2007).

Les recherches récentes qui s'intéressent aux processus de transfert de la valeur ajoutée non financière reconnaissent l'importance d'établir des relations sociales fortes entre les deux parties (ibidem p. 206). Une modalité clé du transfert de la valeur ajoutée entre les deux parties est donc l'existence entre entrepreneurs et capital investisseurs de relations personnalisées de type «intuitu personae»: des relations

\footnotetext{
${ }^{1}$ Dans l'article, nous utiliserons de manière indifférenciée les termes de « dirigeants » et d' « entrepreneurs » car les dirigeants étudiés sont des entrepreneurs, au sens où ils sont actionnaires de leur entreprise, et où ils détectent des opportunités entrepreneuriales (Kirzner 1973) sur lesquelles reposent la croissance et la rentabilité de leur firme.
} 
idiosyncrasiques propres aux personnes qui y sont engagées, à leurs qualités, donnant lieu au développement d'affinités toujours singulières non transposables à d'autres personnes.

Les processus de transfert de la valeur-ajoutée sont qualifiés par Large et Muegge de "moderating factors », c'est-à-dire de facteurs expliquant la transformation des apports potentiels des capital investisseurs (value-added inputs) en apports effectifs (value-added outputs). Dans les travaux existants, on en distingue quatre types (De Clercq et Manigart 2007): la confiance, l'engagement, l'interaction sociale et la convergence de buts. A l'exception de la convergence des buts $^{2}$, les trois autres processus reposent sur des relations personnalisées entre entrepreneurs et capital investisseurs telles que définies précédemment et méritent d'être approfondis.

\subsection{La confiance}

La confiance s'est progressivement imposée dans les recherches sur le capital risque comme un élément important des relations entre les deux acteurs (De Clercq et Manigart 2007). La confiance désigne un état psychologique caractérisé par l'existence d'anticipations positives concernant le comportement d'autrui dans des situations comportant des risques et une interdépendance forte entre les parties. Faire confiance à autrui revient ainsi à accepter d'être vulnérable face aux actions de cet autrui en qui l'on a confiance (De Clercq et Sapienza 2001 ; Boon et Holmes 1991). Par exemple, la confiance dans les membres de l'équipe entrepreneuriale est une question clé pour les capital risqueurs et ce, dès avant la signature du deal car elle permet de pallier le manque d'informations tangibles sur l'entreprise et le caractère peu scientifique de l'évaluation (Sweeting 1991, p. 619). Sapienza (1989) a montré que les capital-risqueurs qui connaissent des succès cherchent à construire des relations de confiance avec les entrepreneurs de leur portefeuille. En effet, la confiance est le vecteur d'une meilleure communication entre les deux acteurs (Sapienza et Timmons 1989 ; Bonnet 2005), elle permet de tirer meilleur profit de la

\footnotetext{
${ }^{2}$ La convergence de buts («goal congruence ») quant à elle a plutôt été analysée comme un élément de nature inter-organisationnelle. Elle désigne le fait que les partenaires de l'échange partagent des croyances similaires (Nahapiet and Ghoshal 1998), des objectifs et des valeurs communs concernant leur relation (Lane et Lubatkin 1998). Elle se perçoit à travers une vision commune concernant l'avenir de l'entreprise ou un accord sur les objectifs prioritaires, financiers ou non (Sapienza et Gupta 1994).
} 
relation, notamment en terme d'échange de connaissances entre les parties (De Clercq et Sapienza 2001) et elle contient souvent la promesse d'une meilleure performance.

Définie comme une présomption, la confiance a principalement été appréhendée à un niveau inter-individuel (e.g. Sheperd et Zacharakis 2001 ; Bonnet 2005) dans le cadre d'interactions entre un entrepreneur et un capital risqueur donnés (De Clercq et Sapienza 2001). Plusieurs éléments d'ordre interpersonnel sous-tendent la construction de cette confiance (Bygrave et Timmons 1991; Sheperd et Zacharakis 2001 ; Sapienza et Korsgaard 1996) : des échanges fréquents et ouverts entre les partenaires ; la présence d'un «fit» (autrement dit, de points de vue, croyances et valeurs partagés); l'existence antérieure de relations personnelles entre investisseur et entrepreneurs ; et, des comportements dans lesquelles une partie signale son engagement en acceptant de se rendre vulnérable face à l'autre partie ${ }^{3}$.

\subsection{L'engagement}

L'engagement désigne l'intensité relationnelle de la coopération entre les partenaires (De Clercq et Manigart 2007, p. 207). Il se manifeste dans des comportements spécifiques reflétant la volonté du partenaire d'investir grandement dans la relation (p. 210). Il correspond aux efforts que font les entrepreneurs comme les investisseurs vis-à-vis de l'autre partie (Gifford 1997). Ces efforts concernent, par exemple, le reporting communiqué par l'entrepreneur ou la mobilisation par le capital risqueur de son réseau de contacts. Chacun des acteurs de la relation s'identifie à l'autre donnant ainsi naissance à une dimension affective et émotionnelle dans leurs échanges. L'engagement se mesure notamment à travers le temps consacré aux échanges (Ehrlich et al. 1994 ; Gorman et Sahlman 1989; Sapienza 1992), ou encore au nombre de contacts entre les partenaires. Ainsi, l'engagement se déploie dans le cadre d'interactions inter-individuelles singulières entre des chargés d'investissement et des entrepreneurs (De Clercq et Fried 2005 ; Jackson III et al. 2012).

Au-delà de la controverse sur les effets bénéfiques de l'engagement sur la performance de l'entreprise, question sur laquelle se sont

\footnotetext{
${ }^{3}$ Un élément d'ordre organisationnel a aussi été identifié comme favorisant l'émergence de la confiance : la mise en place de processus et de procédures «justes » (Sheperd et Zacharakis 2001).
} 
concentrés de nombreux travaux (par exemple, De Clercq et Sapienza 2006), il est intéressant de souligner que le degré d'engagement est très variable selon les investisseurs (Macmillan et al. 1989; Sapienza et Timmons 1989). Trois profils de capital investisseurs sont distingués : ceux avec un engagement faible (profil de «laissez-faire»), ceux au contraire avec un engagement fort («close tracker involvement »), ceux enfin avec un engagement «modéré». Cette variabilité s'explique certes par des variables organisationnelles comme la taille et la diversité du portefeuille du capital risqueur, ou encore l'origine des fonds investis (Fulghieri et Sevilir 2009) mais aussi par des caractéristiques individuelles qui influencent la forme que prend l'engagement. Par exemple, les dirigeants peuvent avoir des perceptions et des souhaits spécifiques concernant le contenu des informations de gestion (leur niveau de détail) à transmettre à leur capital investisseur (Cavelius et Dessertine 2009). Le capital humain des chargés d'investissement (leur formation, leur expérience) détermine également dans une certaine mesure leur capacité à prodiguer des conseils financiers et managériaux aux entreprises de leur portefeuille (Jackson III et al. 2012, p. 344).

\subsection{Les interactions sociales}

Les interactions sociales désignent à la fois l'aspect informel et la force des liens entre les partenaires. Il a été démontré que l'existence de contacts interindividuels forts et personnalisés entre les partenaires accroissait la volonté de chacun de rester engagé dans l'échange avec l'autre sur une période de temps longue (Morgan et Hunt 1994). Un lien positif a aussi été démontré entre l'importance des interactions entre capital risqueur et entrepreneur lors de rencontres «sociales» non strictement professionnelles d'une part et la performance perçue par le capital risqueur des investissements d'autre part (De Clercq et Sapienza 2006). Des contacts sociaux, informels et réguliers peuvent en effet amener l'investisseur à davantage aider l'entrepreneur, pour des raisons autres que purement économiques, et favoriser ainsi le transfert de savoirs complexes tacites (De Clecq et Manigart 2007). Le fait d'avoir prouvé, pour un entrepreneur, sa volonté de développer des relations sociales fortes et ouvertes avec des investisseurs constitue un signal positif lui ouvrant potentiellement l'accès à des financements complémentaires (ibidem). 
Finalement, les travaux existants révèlent que trois des quatre processus permettant le transfert de valeur ajoutée non financière des investisseurs aux entrepreneurs s'appuient sur des relations personnalisées entre les deux acteurs relevant de caractéristiques individuelles. Ils adoptent une approche interindividuelle, reposant sur le recueil des points de vue de capital investisseurs et, dans certains cas, d'entrepreneurs, qu'il serait intéressant de contextualiser. Les capital investisseurs, en effet, travaillent dans des organisations - sociétés de capital investissement - dont ils sont salariés. Pour penser la manière dont ces organisations pèsent sur travail des chargés d'investissement, notamment sur le développement de relations personnalisées avec les entrepreneurs, nous ferons appel à la sociologie de la bureaucratie.

\section{La sociologie de la bureaucratie : la production de règles génériques structurant le travail}

La sociologie de la bureaucratie de Merton (1940), Gouldner (1954), Selznick (1943), et Crozier (1964) a mis en lumière le poids des règles et leur nature dans les organisations «bureaucratiques »

modernes. L'une des caractéristiques fondamentales de ces organisations apparues au tournant $\mathrm{du} \mathrm{XX}^{\text {ème }}$ siècle est en effet l'existence de règles et de procédures impersonnelles, applicables à tous leurs membres, et qui structurent l'activité et les comportements des individus en dehors de leurs caractéristiques individuelles singulières (Crozier 1964; Weber 2003). Dans les organisations bureaucratiques, l'activité est ainsi liée à des règles techniques explicites fixées par écrit (Weber 2003) qui prennent alors par exemple la forme de codes de procédures et de règlements prescrivant les comportements attendus. Si ces règles impersonnelles sont garantes d'une efficience accrue, leurs effets pervers et leurs dangers ont très vite été soulignés dans les travaux de sociologie de la bureaucratie. Déjà en 1921, Weber (2003) s'inquiétait du caractère déshumanisant de ces systèmes organisationnels, tandis que Merton, Gouldner et Selznick ont questionné le lien entre la standardisation de l'individu ainsi engendrée et l'efficacité de ces organisations. Merton (1940) a notamment souligné combien la discipline et le comportement standardisé prônés par ces organisations créaient un fossé entre le bureaucrate et son public. Il ressort finalement des études classiques de 
sociologie de la bureaucratie que les règles génériques caractéristiques des organisations modernes - y compris celles qui sont plus éloignées de l'idéal type de la bureaucratie - concourent à développer des relations relativement dépersonnalisées entre leurs membres et leur public.

Dans certains de ses prolongements, cette sociologie a mis l'accent sur la manière dont ces règles pouvaient être contournées, et donc peu appliquées, par les acteurs (e.g. Crozier et Friedberg 1977). L'exception à la règle conforte la position de ceux qui peuvent s'en affranchir et a un impact sur la distribution du pouvoir. Les règles sont alors autant des ressources que des contraintes et participent à l'orientation des comportements dans les organisations mais parfois avec des effets inattendus. Le succès relatif des règles est alors un effet de normalisation des comportements sans prescription absolue. Ces prolongements ont aussi enrichi la compréhension de la nature de ses règles en distinguant les règles formelles des règles informelles (ibidem), les secondes pouvant s'apparenter à des normes partagées et considérées comme «allant de soi » (Goffman 1973 ; Meyer et Rowan 1977) par les membres de l'organisation. C'est ainsi qu'en plus des règles techniques, des normes de comportements informelles mais malgré tout prescriptives viennent également encadrer les relations interpersonnelles en agissant sur les liens de confiance ou d'engagement. Sans prescrire précisément les actions, ces normes promeuvent des habitudes de travail qui sont ensuite déclinées, sans être systématiquement contournées, par les acteurs. Au total, se pose la question de savoir comment s'articulent les règles produites par les organisations, applicables à tous leurs membres, et la dimension personnalisée de la relation investisseur-entrepreneur précédemment mise en lumière. Une originalité de cet article est de montrer comment le respect de règles de type bureaucratique permet aux capital investisseurs de poursuivre collectivement la mise en place de relations personnalisées avec les entrepreneurs.

\section{Méthodologie et caractéristiques de l'étude de cas}

Les bases empiriques de ce travail reposent sur une enquête de terrain réalisée en 2010 auprès de l'ensemble des directeurs de participation d'une société régionale d'investissement en capital, dénommée dans ces pages Capital-Invest (un pseudonyme). Cette 
section présente les raisons qui justifient le choix d'une étude de cas unique, les caractéristiques de l'entreprise étudiée et les modalités de recueil et de traitement des données.

\subsection{Design de la recherche}

Pour étudier la manière dont les capital investisseurs construisent des relations interpersonnelles avec les entrepreneurs, l'analyse a porté sur les mécanismes organisationnels qui encadrent les pratiques de travail des directeurs de participation («DP») de Capital-Invest. D'un point de vue conceptuel, la perspective adoptée s'apparente à celle de la sociologie de l'action collective (e.g. Crozier et Friedberg 1977) qui replace l'individu dans son contexte organisationnel pour en étudier le comportement au travail. Les investisseurs ne sont alors pas appréhendés comme des individus décontextualisés, isolés ou engagés dans une relation inter-individuelle dyadique avec un entrepreneur mais comme des salariés de Capital-Invest, inscrits dans une organisation qui leur fixe des règles, des procédures à respecter, dans le cadre d'une pratique professionnelle collective et organisée. Nous nous intéressons alors à la manière dont l'organisation du travail et le collectif pèsent sur la construction de relations entre investisseurs et entrepreneurs.

Pour aborder cette question, une recherche fondée sur une étude de cas unique a été mise en œuvre. Cette méthodologie de recherche est en effet adaptée pour appréhender des mécanismes processuels, interdépendants et dynamiques dans le temps, et répondre à une question sous forme de «comment » (Eisenhardt et Graebner 2007). Il s'agit ici d'une étude de cas «descriptive » (Yin 1994) qui analyse en profondeur les pratiques des investisseurs au travail. L'unité d'analyse est donc l'organisation.

Le choix du cas a été guidé par différents critères. L'opportunité qui a été offerte aux auteurs de rencontrer la totalité des DP de la société de capital investissement a été déterminante. Elle a rendu possible, à partir de l'analyse des pratiques individuelles des DP, la mise en lumière des éléments organisationnels intervenant, au niveau d'une société de capital investissement, dans l'émergence de relations entrepreneursinvestisseurs. Elle a ainsi permis d'identifier de manière fiable des pratiques collectives de travail à la fois formelles et informelles. L'ancienneté de la société étudiée et sa taille relativement importante ont aussi présidé au choix du cas. Ces éléments garantissaient en effet 
que les pratiques de travail seraient relativement stabilisées, et donc identifiables, et que les relations avec les entrepreneurs auraient fait l'objet d'apprentissages individuels et collectifs. Enfin, une autre caractéristique renforçait l'intérêt du cas : Capital-Invest est une société d'investissement qui a su s'imposer sur son marché comme leader et, donc, au sein de laquelle on pouvait s'attendre à identifier certaines «bonnes pratiques ».

\subsection{Recueil et analyse des données}

Onze entretiens semi-directifs d'une durée moyenne d'une heure et demie - enregistrés et intégralement retranscrits - ont été conduits auprès de tous les DP de Capital-Invest ${ }^{4}$. Les entretiens furent conduits sur la base d'un guide d'entretien ( $c f$. annexes) qui n'a pas évolué, en dehors de quelques reformulations, au cours de la phase d'enquête. Les questions ainsi répliquées à l'identique auprès de nos différents interlocuteurs ont permis d'identifier et de recouper les pratiques de travail des DP aux différentes phases de la vie des participations que sont la sélection, le suivi et la sortie. C'est ce découpage entre les différentes phases de la relation qui fut retenu afin d'organiser la présentation des éléments empiriques ( $c f$. section 4) dans le but d'illustrer la diversités des règles organisationnelles mises en œuvre en soutien des relations personnelles tissées avec les entrepreneurs. Les entretiens ont de plus fait l'objet d'un travail de codage systématique en fonction de catégories analytiques telles que «la construction de la confiance » et « la sélection des participations » correspondant chacune à un thème particulièrement saillant empiriquement. Ce travail a permis de faire émerger les mécanismes organisationnels mobilisés dans les relations avec les directeurs de participations.

Les données recueillies au cours des échanges ont également été complétées par l'analyse des rapports d'activités publiés entre 2004 et 2009 et par une observation non participante à une réunion d'investissement de Capital-Invest. Sur la base des matériaux empiriques collectés, une analyse intermédiaire a été réalisée sous la

\footnotetext{
${ }^{4}$ Les dix DP de Capital-Invest ont été interviewés, suite à un premier entretien conjoint avec le directeur et le directeur adjoint. Ces derniers ont par la suite également été interviewés individuellement au titre de leur activité, demeurée importante, de directeur de participation. Ces entretiens sont intégrés aux dix entretiens de DP dont il est fait mention dans la suite de l'article (notamment dans le Tableau 3).
} 
forme d'une monographie. La restitution, à l'ensemble des DP de Capital-Invest, des premiers résultats de l'équipe de recherche a permis de les valider.

Le processus d'analyse des données a été gouverné par une logique de comparaison systématique entre les entretiens. La logique de l'étude de cas «impliqu[ant] de faire des inférences à partir de similarités ou de différences de caractéristiques entre des paires » (Langley et Royer 2006 , p. 82, traduit par nous), nous a notamment conduit à comparer les pratiques de travail des DP anciennement et nouvellement recrutés ainsi que celles des DP aux expériences professionnelles contrastées sans révéler de différence majeure. Les données recueillies en entretien ont ainsi été systématiquement rapprochées les unes des autres afin de reconstruire avec fiabilité les caractéristiques du système organisationnel encadrant le travail des DP.

\subsection{Caractéristiques du cas}

Capital-Invest est une société de capital investissement régionale créée au début des années 1980 dans le cadre d'une politique nationale de développement du capital investissement. Disposant au départ d'une structure au capital diversifié, elle est progressivement devenue la filiale d'une grande banque nationale qui offre uniquement du capital développement et du capital transmission.

Au moment de l'enquête, Capital-Invest occupait une place majeure sur ses marchés. Avec plus de 80 millions d'Euros investis en 2008 dans 27 participations, la société comptait pour environ $10 \%$ du marché du capital développement et capital transmission sur les six régions où se concentrait son activité. Elle disposait en outre avec 156 sociétés accompagnées du plus important portefeuille de participations parmi les sociétés de capital investissement régionales concurrentes.

Capital-Invest présente plusieurs spécificités liées à son statut juridique de «société de capital risque $»(« \mathrm{SCR} »)^{5}$. En effet, de manière à promouvoir le financement des PME, les SCR sont exonérées de l'impôt sur les sociétés pour les revenus provenant des produits nets, des plus-values réalisées sur la vente d'action ainsi que des revenus de placements en «attente d'investissement». Pour bénéficier de ces

\footnotetext{
${ }^{5}$ Le statut des SCR a été créé par la loi 85-695 du 11 juillet 1985. Il est venu se substituer au statut d'Instituts Régionaux de Participations (IRP), qui était celui de Capital-Invest à sa fondation.
} 
avantages, les SCR doivent néanmoins prendre des participations inférieures ou égales à $40 \%$ du capital des entreprises et investir sur fonds propres et sans imposer de terme temporel défini à l'avance à leurs participations. En l'absence de clause de liquidité, la durée des investissements de Capital-Invest est plutôt supérieure à celle des fonds d'investissement classiques ${ }^{6}$ : si la durée moyenne est de 6 ans, il est courant que, par le biais d'investissements successifs, des entreprises restent au portefeuille de Capital-Invest pendant 15 ou 20 ans. Le Tableau 1 présente les principales caractéristiques de l'activité de Capital-Invest.

Tableau 1 - Résumé des principales caractéristiques de Capital-Invest

\begin{tabular}{|l|l|}
\hline Statut juridique & $\begin{array}{l}\text { SCR, devenue captive à la fin des années } \\
2000\end{array}$ \\
\hline Capital investi & Entre 500 K€ et 15 000 K€ \\
\hline Implication dans les syndications & Peu fréquente et peu désirée \\
\hline Représentation au conseil d'administration & $\begin{array}{l}\text { Possible mais pas exigée. Un siège à un } \\
\text { comité d'information est préféré. }\end{array}$ \\
\hline Temps d'investissement moyen & $\begin{array}{l}\text { Environ 6 ans mais parfois reconduit } \\
\text { plusieurs fois avec une même société }\end{array}$ \\
\hline Nombre d'investissements en portefeuille & $\begin{array}{l}\text { Plus de 150 au moment de l'enquête } \\
\text { (environ 500 entreprises accompagnées } \\
\text { depuis la création de la société) }\end{array}$ \\
\hline
\end{tabular}

Capital-Invest présente malgré tout des similarités avec l'ensemble des sociétés d'investissement captives - notamment, une préoccupation moindre que les capital investisseurs indépendants pour la durée effective de leurs investissements (Desbrières et Broye 2000). Elle partage également avec la plupart des capital investisseurs régionaux un intérêt accru pour préserver l'ancrage territorial de leurs participations (Stévenot-Guéry 2007).

\section{Les mécanismes organisationnels de construction de relations interpersonnelles}

Cette partie, consacrée aux résultats de l'étude de cas, montre comment l'organisation du travail de Capital-Invest oriente les DP vers la construction de relations interpersonnelles avec les entrepreneurs.

\footnotetext{
${ }^{6}$ A savoir les fonds d'investissement gérés par des sociétés de gestion, du type Fonds Commun de Placement à Risques (FCPR) qui recueillent des fonds auprès d'investisseurs et les allouent au financement de projets sur une durée légalement limitée.
} 
Les mécanismes organisationnels qui encadrent et modèlent les pratiques de travail des DP sont présentés en suivant chacune des étapes de l'activité d'investissement, à savoir: son identification et sa sélection (4.1.), son évaluation et sa structuration (4.2.) et son suivi (4.3.).

\subsection{Les règles communes d'identification et de sélection permettant une personnalisation de la relation}

Nos données mettent en lumière l'existence de modalités de construction des relations interpersonnelles dès les premiers contacts avec un entrepreneur. La construction très en amont de relations privilégiées entre les DP et les entrepreneurs obéit à un double enjeu : contenir la concurrence des autres sociétés de capital investissement et se concilier l'intérêt de dirigeants repérés comme intéressants mais parfois réticents à l'idée de faire entrer une institution financière dans le capital de leur entreprise. Plusieurs éléments constitutifs de l'organisation du travail au sein de Capital-Invest facilitent, pour les DP, la création de liens interpersonnels : les consignes de prospection qui leur sont imposées (4.1.1.) ainsi que les pratiques de GRH et les dispositifs qui soutiennent l'octroi de conseils gracieux aux entrepreneurs (4.1.2.)

\subsubsection{Une organisation stricte du travail de prospection à l'origine d'interactions étroites}

$\mathrm{Si}$ nous avons choisi de traiter conjointement les phases d'identification et de sélection des affaires (désignées ci-après sous le terme général d'«identification» des prospects), c'est qu'elles sont concomitantes dans le cas étudié. En effet, à l'inverse du cas de figure classiquement repris dans la littérature (Tyebjee et Bruno 1984 ; Petty et Gruber 2011) mettant en scène des capital risqueurs appelés à choisir entre des dossiers qui leur sont soumis, les cibles intéressantes pour Capital-Invest doivent le plus souvent être approchées afin d'échapper à une concurrence accrue. Dans ce contexte, la définition formelle du travail des DP comprend une intense activité de «séduction» des entrepreneurs qui vise à créer des liens interpersonnels précoces.

La phase d'identification se caractérise tout d'abord par l'application, par les DP, d'un ensemble de critères d'investissement 
prédéfinis assez simples. Ces critères sont résumés dans le Tableau 2. Ils dessinent comme entreprise cible une affaire rentable, indépendante, située dans le Grand Ouest de la France et dirigée par un chef d'entreprise majoritaire, qui a démontré par le passé sa capacité à faire croître l'entreprise. Ces critères positionnent Capital-Invest sur le marché des PME/PMI régionales rentables, porteuses d'une faible incertitude (Knight 1921) ${ }^{7}$ et donc au risque commercial et technologique minimal.

Tableau 2 - Critères d'investissement de Capital-Invest

\begin{tabular}{|l|l|}
\hline Secteur géographique privilégié & Le grand ouest \\
\hline $\begin{array}{l}\text { Secteurs économiques } \\
\text { privilégiés }\end{array}$ & $\begin{array}{l}\text { Aucun, mais méfiance à l'encontre des } \\
\text { nouvelles technologies }\end{array}$ \\
\hline Taille des entreprises cibles & Entre 5 M€ et 100 M€ de CA \\
\hline $\begin{array}{l}\text { Performance passée de } \\
\text { l'entreprise cible }\end{array}$ & Rentable depuis quelques années \\
\hline $\begin{array}{l}\text { Structure du capital de } \\
\text { l'entreprise cible }\end{array}$ & Un propriétaire-dirigeant majoritaire \\
\hline
\end{tabular}

Aux dires des DP interrogés, les entreprises susceptibles de correspondre à ces critères sont plutôt rares : le «vivier» serait de quelques dizaines d'entreprises par département couvert soit environ 2000 entités sur l'ensemble du Grand Ouest. En raison de cette rareté, l'organisation Capital-Invest assigne à ses DP une mission de rapprochement intense avec les prospects - qui occupe, selon les estimations des intéressés, $60 \%$ de leur temps. Ce travail d'identification est encadré par plusieurs règles et dispositifs organisationnels qui engendrent des interactions précoces et répétées avec les dirigeants d'entreprises cibles. La division du travail obéit tout d'abord à une logique géographique : chaque DP est responsable d'un territoire qu'il doit prospecter en profondeur. Une première étape vise à obtenir des informations permettant de détecter de possibles opérations sur le capital d'entreprises remplissant les critères précédemment décrits. Elle consiste en une analyse régulière des informations publiques contenues dans les quotidiens et magazines économiques, ainsi que dans les publications émanant d'organismes locaux diffusant des informations sur l'activité économique comme les bulletins d'informations publiés par les CCI, les associations professionnelles ou encore les clubs patronaux.

\footnotetext{
7 Knight (1921) distingue l'incertitude (non probabilisable) des risques (probabilisables).
} 
Ensuite, les DP entretiennent en permanence leurs relations avec un ensemble de professionnels proches du tissu économique régional : les experts-comptables, les commissaires aux comptes, les avocats d'affaires. Ces intermédiaires sont régulièrement approchés afin d'obtenir un contact avant même l'expression d'un besoin de financement. Ces étapes préliminaires visent ici avant tout à obtenir un rendez-vous en face-à-face avec le dirigeant. Le but des investisseurs de Capital-Invest est de fréquenter personnellement les dirigeants au plus tôt afin d'apprendre à les connaître et de se faire connaître. Cette organisation permet d'intégrer rapidement de nouveaux DP qui se voient par exemple assigner une zone géographique particulière où reproduire les pratiques en œuvre ailleurs dans le groupe.

Le premier rendez-vous avec l'entrepreneur permet de faire un tri entre (i) les entreprises pour lesquelles une demande de financement en capital existe pouvant donner lieu à une prise de participation à brève échéance (dans l'année ou les mois à venir), et (ii) celles qui sont intéressantes, mais dépourvues de tel besoin de financement à court terme. Tandis que les premières rentrent en phase d'évaluation (cf. 3.2), les secondes font l'objet d'un travail systématique de veille et de « séduction » de leur dirigeant par les DP de Capital-Invest.

L'enjeu pour Capital-Invest est que ses DP aient l'assurance d'être informés par le dirigeant de tout besoin de financement et qu'ils puissent se mettre en situation d'être choisi plutôt qu'un concurrent pour une prise de participation. La personnalisation de la relation avec l'entrepreneur est un moyen pour atteindre ce but.

"Si je discute avec le dirigeant depuis 10 ans, à prix égal il me choisit (rires) voilà ! [...] A nous d'être suffisamment présents pour éviter la plus grosse compétition possible.» (DP 6)

$\mathrm{Au}$ moment de l'enquête, selon les estimations de nos interlocuteurs, $90 \%$ des opérations conduites par Capital-Invest se faisaient sans mise en concurrence avec d'autres sociétés de capital investissement. Pour cela, les DP sont encouragés par Capital-Invest à consacrer le temps et les ressources nécessaires de manière à saisir et multiplier les occasions d'interagir directement avec les dirigeants. L'organisation, trois à quatre fois par an d'événements de rencontre, appelés «journées entrepreneurs », entre des entrepreneurs de sociétés dans lesquelles Capital-Invest a investi et des entrepreneurs cibles qui n'ont pas encore ouvert leur capital, est un autre moyen d'entrer en contact avec les dirigeants repérés et de les «séduire». Lors de ces 
«journées » les témoignages de dirigeants du portefeuille sur leur expérience avec Capital-Invest permettent de renouveler le contact interpersonnel avec les prospects et «c'est une façon d'apprivoiser certains interlocuteurs qui peuvent être un peu réticents » (DP 7). Ces rencontres, entièrement orchestrées et planifiées par Capital-Invest sont autant d'occasions de nourrir des échanges informels entre ses employés, les clients actuels et les prospects.

\subsubsection{Une institutionnalisation du conseil gracieux personnalisé}

En matière de contenu, cette relation personnalisée entre DP et entrepreneurs se nourrit de l'octroi régulier, par les premiers, de conseils gratuits aux seconds, et de mise en relation avec des partenaires intéressants. Autrement dit, c'est à travers l'institutionnalisation par Capital-Invest d'une offre non marchande de conseils et de contacts pertinents que se construit une relation privilégiée avec les entrepreneurs. Cette institutionnalisation est soustendue par deux éléments organisationnels : les pratiques de GRH de Capital-Invest et des dispositifs de formalisation/mutualisation des connaissances sur les entreprises cibles. Concernant le premier point, la politique de recrutement et le faible turn-over chez Capital-Invest sont autant de facteurs qui permettent aux DP de disposer d'une connaissance très fine des PME/PMI régionales et de leurs problématiques, ainsi que de relations fortes avec des partenaires pertinents pour les dirigeants (autres entrepreneurs, experts-comptables, avocats, banquiers, etc.). Les DP recrutés disposent en général d'une expérience professionnelle antérieure dans le financement d'entreprises régionales ( $c f$. Tableau 3), souvent dans la même région que CapitalInvest. Les départs étant très rares (depuis 10 ans, un seul DP a quitté Capital-Invest), l'expérience des DP est donc très élevée. Au moment des entretiens, la durée moyenne de présence chez Capital-Invest est de 12,8 ans.

Tableau 3 - Ancienneté des capital investisseurs de Capital-Invest

\begin{tabular}{|c|c|l|}
\hline $\begin{array}{c}\text { Directeur de } \\
\text { participations }\end{array}$ & $\begin{array}{c}\text { Ancienneté } \\
\text { Capital-Invest* }\end{array}$ & \multicolumn{1}{c|}{ Poste précédent } \\
\hline DP 1 & 7 & $\begin{array}{l}\text { 30 ans dans le capital investissement } \\
\text { régional }\end{array}$ \\
\hline DP 2 & 24 & Inconnu \\
\hline DP 3 & 22 & $\begin{array}{l}\text { 12 ans chez un capital investisseur } \\
\text { régional, concurrent de Capital-Invest }\end{array}$ \\
\hline
\end{tabular}




\begin{tabular}{|l|l|l|}
\hline DP 4 & 11 & 3 ans, Cabinet audit (audits d'acquisition) \\
\hline DP 5 & 14 & $\begin{array}{l}10 \text { ans chez un capital investisseur } \\
\text { régional, concurrent de Capital-Invest }\end{array}$ \\
\hline DP 6 & 6 & $\begin{array}{l}\text { 2 ans, Branche finance d'entreprise d'une } \\
\text { filiale régionale d'une grande banque }\end{array}$ \\
\hline DP 7 & 7 & $\begin{array}{l}7 \text { ans, analyste financier en société de } \\
\text { bourse }\end{array}$ \\
\hline DP 8 & 6 & $\begin{array}{l}5 \text { ans, Branche finance d'entreprise d'une } \\
\text { filiale régionale d'une grande banque }\end{array}$ \\
\hline DP 9 & 6 & $\begin{array}{l}\text { 5 ans chez un capital investisseur régional, } \\
\text { concurrent de Capital-Invest }\end{array}$ \\
\hline DP 10 & 25 & $\begin{array}{l}12 \text { ans en Banque régionale, relations } \\
\text { PME-PMI }\end{array}$ \\
\hline
\end{tabular}

* calculée avec 2012 comme année de référence.

L'existence au sein de Capital-Invest de procédures de formalisation et de mutualisation des connaissances sur les entreprises cibles constitue un mécanisme de construction collective d'une expertise et d'un réseau de grande valeur pour les entrepreneurs. Ainsi, toute visite au sein d'une entreprise donne lieu à la rédaction d'une note qui vient incrémenter une base informatique de prospection. En plus d'être outillé, le partage des connaissances est ritualisé au travers de la réunion commerciale hebdomadaire. Tous les DP ont ainsi l'obligation d'assister à ces réunions où sont discutés collectivement, pour chaque prospect, les points qui posent question ainsi que les informations concernant le dirigeant, le secteur ou les concurrents.

Le système de rémunération des DP constitue un autre élément favorisant le partage de connaissances. La rémunération variable versée sous forme de primes annuelles - est entièrement collective, calculée sur la base des résultats du portefeuille dans son ensemble. Ce système permet de réduire les risques de mauvaise coopération au sein de la population des investisseurs en encourageant l'entraide dans le but de « signer des deals».

Pour conclure, soulignons que la personnalisation de la relation avec l'entrepreneur, à ce stade de l'activité d'investissement, obéit aux deux enjeux des phases d'identification et de sélection. Il s'agit tout d'abord de capter une demande de financement en partie cachée (identification), en «séduisant» le dirigeant afin d'écarter la concurrence. L'objectif est aussi d'apprécier le risque managérial (sélection) - lié au dirigeant et à sa personnalité. Les premiers contacts avec ce dernier sont l'occasion d'apprécier finement et de manière tacite (1) la capacité managériale de l'entrepreneur et (2) ses capacités 
de coopération avec l'investisseur. Il s'agit de s'assurer que le dirigeant comprendra et acceptera les contraintes et les objectifs de ses investisseurs, notamment leur volonté de réaliser un profit financier. Pour ce faire, les DP déploient un jugement fortement intuitif, reposant sur des signaux qui émergent de l'interaction avec le dirigeant dès la phase d'identification. Les moyens mis en ouvre par Capital-Invest dans le but de soutenir le développement de relations interpersonnelles rapprochées avec les dirigeants mettent ainsi les DP en position de repérer les signaux de volonté de coopération évoqués par De Clercq et Sapienza $^{8}$ (2001).

\subsection{Normer la décision d'investissement tout en préservant la personnalisation du lien}

Si les critères d'évaluation utilisés par les capital investisseurs ont été finement étudiés (par exemple, Desbrières et Broye 2000), peu d'attention a été accordée à la dimension proprement organisationnelle de la prise de décision, comme en témoigne la récente recension de Petty et Gruber (2011). Notre analyse révèle qu'à travers l'organisation collective du travail lors des phases d'évaluation et de structuration des deals s'opère un travail d'objectivation d'une relation jusque là essentiellement personnalisée (4.2.1.). Cette logique de dépersonnalisation ne marque pourtant pas la fin des efforts de personnalisation de la relation avec les entrepreneurs du fait notamment du maintien de mécanismes promouvant le caractère interpersonnel des échanges (4.2.2.).

\subsubsection{La bureaucratisation de la décision d'investir : dépersonnaliser pour réduir les risques}

Le processus organisationnel de prise de décision d'investissement, qui comprend les phases d'évaluation et de structuration de l'investissement, vise à minimiser les risques de sélection adverse grâce à une prise de distance imposée par Capital-Invest à ses DP. Le détail de ce processus étant présenté en annexe, nous nous concentrons ici sur

\footnotetext{
${ }^{8}$ Certains signaux agissent comme des critères éliminatoires : par exemple, un dirigeant qui «oublie » de fournir des informations ou qui se montre désinvolte à l'égard de la gestion opérationnelle, cessera d'intéresser Capital-Invest. A l'inverse, un entrepreneur qui se montre attentif aux suggestions et remarques et qui démontre son souhait d'échanger aura toute les chances d'intéresser les DP.
} 
la mise en valeur des éléments qui dans ce processus participent à la dépersonnalisation d'un lien que les phases précédentes d'investissement visaient à personnlaliser. Tout d'abord, l'évaluation et la structuration d'un investissement au sein de Capital-Invest sont des processus collectifs qui impliquent obligatoirement tous les DP de la société. Les procédures mises en place pour évaluer la qualité des projets d'investissement ont pour but de garantir l'objectivité du jugement des investisseurs, menacés par la tendance naturelle à «tomber amoureux » du dossier sur lequel ils travaillent depuis des mois voire des années.

"C'est aussi pour ça qu'on a mis en place des réunions commerciales, parce que ça nous permet... quand on est, enfin la personne qui s'investit sur le dossier, elle a par définition moins de recul ou d'objectivité sur son dossier puisqu'elle s'est impliquée, elle a rencontré le dirigeant, elle est la seule à l'avoir fait, c'est essentiel mais pour le coup très subjectif, donc d'échanger en amont avec l'équipe qui, par définition, est objective sur un dossier qu'elle découvre et, pour lequel elle n'a pas rencontré le dirigeant, ça permet justement soit de se poser de nouvelles questions, soit de refermer un dossier... » (DP 4)

Cette mise en commun de la décision, imposée par la direction, vise à accroître la qualité du jugement en le faisant reposer sur des appréciations plus objectives. Les réunions commerciales hebdomadaires constituent la première de ces procédures. A travers l'examen de chaque dossier, de manière répétée lors de réunions successives, sont identifiés et discutés les points qui posent question, à éclaircir avec le dirigeant et ses partenaires ou à préciser par les DP. Ces rencontres formalisées sont le lieu d'exercice, à travers les questions posées et les échanges qui s'ensuivent, d'un regard critique par les pairs sur le projet, visant à déceler les risques qui ont pu être négligés. Ensuite, si le système de rémunération variable collective entre DP incite au partage des connaissances entre investisseurs, c'est surtout le travail en binôme, c'est-à-dire l'obligation de travailler dans cette phase à deux DP sur un même dossier, qui encourage la mutualisation des connaissances, le regard critique et le débat contradictoire. Au final, l'aval pour un investissement ne peut-être obtenu que par une décision majoritaire des DP en faveur du projet. Cet engagement revêt un certain formalisme et avalise définitivement l'engagement de Capital-Invest au coté d'une entreprise et de son dirigeant. 


\subsubsection{Les mécanismes de maintien du caractère personnalisé de la relation}

Malgré ces mesures en faveur d'une prise de distance par rapport aux dossiers suivis, les procédures mises en œuvre lors des phases d'évaluation et de structuration préservent le caractère personnalisé des relations avec les entrepreneurs qui, comme on l'a vu, permettent d'écarter la concurrence. Plusieurs éléments concourent à rendre imperceptibles pour l'entrepreneur la collectivisation et la formalisation du travail d'évaluation. Pendant toute cette phase, le DP initial continue d'être l'interlocuteur principal du dirigeant et cela même s'il doit choisir un collègue pour former le binôme qui structurera l'offre de financement lors des débats internes. La procédure collective organisée d'évaluation et de construction d'une offre reste alors relativement invisible pour le dirigeant. Elle laisse penser au dirigeant qu'il aura à ses côtés un DP qui ne sera pas un simple exécutant de directives de Capital-Invest mais un alter ego, capable de prendre des décisions autonomes et, donc, de l'assister.

"Quand on a réussi à convaincre un dirigeant d'entrer dans le capital de son entreprise c'est plus par sa façon d'être que par sa façon de faire. Donc, je pense que l'employeur apporte beaucoup dans la mesure où il participe à la sérénité du directeur de participations, qu'il l'épanouisse, et c'est là qu'il le met en situation de décider - même s'il y a des comités à respecter - mais le prospect il sent bien tout de suite s'il a affaire à quelqu'un qui va décider, qui va l'assister... » (DP 8)

L'arrivée d'une tièrce personne en charge d'apprécier la qualité du projet ne vient donc pas remettre en cause le principe d'un relatif effacement de l'organisation aux yeux du dirigeant. Ensuite, si les procédures et la formalisation du travail existent, elles restent suffisemment légères pour permettre que les décisions d'investissement soient prises en quelques semaines, tout au plus en deux à trois mois. Les documents produits par Capital-Invest qui sous-tendent la décision d'investir, notamment les notes et les rapports d'investissement, restent relativement courts ( 3 à 4 pages pour les notes intermédiaires, une vingtaine pour le rapport sur lequel se prend la décision) ce qui permet des délais de traitement raccourcis. Fait caractéristique chez CapitalInvest, la procédure de due diligence sert non pas à fonder le jugement et la décision d'investir (ou pas) dans l'entreprise, mais à s'assurer qu'aucun élément n'a été ignoré qui pourrait invalider la décision déjà 
prise et, donc, à valider ex post cette décision. La décision d'investissement est en effet prise avant que la due diligence ne soit achevée et que les documents d'analyse réalisés par des tiers extérieurs ne parviennent à Capital-Invest. La primauté est en effet donnée aux échanges verbaux informels entre les capital investisseurs plutôt qu'au respect de procédures d'audits financiers et juridiques potentiellement lourdes.

Enfin, une règle informelle collectivement partagée par les investisseurs régit le travail dans la phase de structuration: la négociation avec l'entrepreneur doit rester harmonieuse et dépourvue de tensions majeures, de manière à ne pas entacher la qualité de la relation interpersonnelle.

«La phase de négociation ne doit pas laisser de trace, elle ne doit pas être tendue, elle ne doit pas être... on ne doit pas se déchirer sur un prix, sur une valo, souvent ça laisse des traces. Cette espèce de discussion lorsqu'elle est mal menée, elle laisse des traces [...] assez longtemps, durables dans la vie du partenariat. » (DP 7)

Cette volonté amène les DP à externaliser l'évaluation des aspects qui pourraient poser problème en faisant appel à des partenaires extérieurs.

\subsection{Les règles d'un suivi personnalisé des investissements sur le temps long}

Chez Capital-Invest trois types de règles encadrant le travail des DP rendent possible en pratique le développement de relations interpersonnelles avec les dirigeants en phase de post-investissement. Tout d'abord il sera question de l'engagement du DP auprès de l'entrepreneur qui varie dans le temps suivant une règle simple: l'attitude par défaut s'apparente au laisser-faire mais toute demande de soutien émise par l'entrepreneur doit recevoir une réponse attentive et diligente (4.3.1). A cela s'ajoute la définition stricte et circonscrite des champs sur lesquels les investisseurs doivent prodiguer leurs conseils (4.3.2). Enfin, le comportement de ces derniers doit se trouver empreint de modestie et d'adaptabilité (4.3.3). Ces trois règles sont informelles, au sens où elles ne sont ni inscrites ni diffusées dans un quelconque document officiel. Elles correspondent à des normes de comportement intégrées par l'ensemble des DP, et soutenues par l'organisation du travail établie chez Capital-Invest. 


\subsubsection{Une attitude par défaut de "laisser faire » couplée à une grande réactivité}

En phase de post-investissement les DP de Capital-Invest ont pour norme de comportement la mise en oeuvre d'un engagement faible de type «laissez-faire» (Macmillan et al. 1989). Les interactions avec les entrepreneurs se limitent alors aux rencontres réglementaires formelles prévues par les statuts ou par des dispositions propres au pacte d'actionnaires. Lors de ces échanges, les investisseurs acceptent pour l'essentiel sans mot dire les décisions du dirigeant. Ils laissent à ce dernier toute latitude et lui renvoient volontiers l'image qu'ils perçoivent comme attendue d'eux : "S'il n'y a pas de vrai problème à soulever, alors il faut dire amen à tout» (DP 6). Cette attitude se traduit aussi par des exigences relativement faibles en matière de contrôle qui est adapté au style et aux habitudes singulières du dirigeant : un reporting est exigé, mais les formes que l'entrepreneur lui donne restent largement à sa discrétion.

"Une des forces [de Capital-Invest], c'est de pouvoir selon les dossiers, selon les situations, s'adapter un peu en fonction aussi selon les attentes du dirigeant, adapter son suivi, tout n'est pas figé dans un formalisme. » (DP 4)

Capital-Invest impose également à ses DP de toujours répondre à une demande explicite de soutien émanant d'un dirigeant du portefeuille. Pareilles sollicitations déclenchent alors pour un temps limité un engagement non plus faible mais «rapproché ». Au total, cette double attitude de « laissez-faire » et de diligence par rapport aux demandes des entrepreneurs, constitue une modalité de construction de la confiance entre les partenaires, en évitant que les entrepreneurs n'aient le sentiment d'être ennuyés de manière superflue par leurs investisseurs.

Notre enquête empirique révèle ainsi que, contrairement à ce que laisse entendre une partie de la littérature (par exemple, Macmillan et al. 1989), le niveau d'engagement des DP ne constitue pas une caractéristique stable. Loin de se résumer à des profils types, il est au contraire chez Capital-Invest une réalité dynamique variant très fortement en intensité selon les moments considérés. La variabilité de l'engagement et les règles de déclenchement d'un engagement exceptionnel plus rapproché constituent pour les capital investisseurs un moyen d'allocation de leur temps entre les 14 participations qu'ils 
suivent en moyenne. Elles leur permettent de se référer à des règles communes d'arbitrage pour régler l'intensité d'un engagement par essence chronophage. En complément des travaux qui tendent à démontrer qu'il y a une taille de portefeuille au delà de laquelle le capital investisseur ne peut plus assurer un suivi réel (Jackson III et al. 2012), nos recherches mettent en valeur le fait que les capital investisseurs se dotent de normes permettant de doser leur engagement pour concilier des enjeux contradictoires : limiter le temps dédié aux relations avec l'entrepreneur, tout en ne déplaisant pas à celui-ci et en restant vigilant pour contrôler l'évolution de l'entreprise.

\subsubsection{Des conseils circonscrits et « sur mesure » pour garantir sa légitimité}

La seconde règle informelle qui régit l'entretien des relations interpersonnelles entre les partenaires consiste, pour les capital investisseurs de Capital-Invest, à limiter le spectre de leurs conseils à deux thématiques: le financement et la stratégie générale de développement de l'entreprise. Les conseils financiers concernent la mise en oeuvre d'indicateurs de gestion pertinents (comme l'EBITDA) ou de techniques financières particulières (par exemple la déconsolidation d'actifs immobiliers), mais aussi l'accompagnement des entrepreneurs pour bien négocier avec les acteurs financiers. Quant aux échanges stratégiques, ils touchent souvent à des thématiques de croissance externe. Il s'agit là de deux domaines d'expertise que les DP acquièrent à travers leur longue expérience dans le métier et les dispositifs de mutualisation des connaissances au sein de l'organisation (cf. 4.1.2. et 4.2.1.). L'expérience de Capital-Invest dans plus de 500 entreprises régionales leur fournit ainsi une base de références et d'exemples portant sur les choix financiers et le développement des PME qui s'avère très pertinente. Il ressort qu'un moyen de se prémunir de tensions et d'éventuels comportements opportunistes est pour les DP de se montrer proches des dirigeants dans la phase de suivi, notamment en prodiguant des conseils perçus comme pertinents.

"Ce n'est pas parce qu'on a apporté de l'argent à un temps « 0 » $q u$ 'on est légitime. On a réussi un partenariat et on gagne la confiance du dirigeant mais il faut qu'on donne autre chose. Si on ne fait qu'apporter de l'argent, on n'est pas beaucoup considéré. » (DP 3) 
Le choix de Capital-Invest de répondre très précisément à des questions soigneusement choisies traduit la volonté des capital investisseurs de construire et préserver une relation de proximité avec les dirigeants sans pour autant s'exposer à devoir répondre à des demandes excessives. Ces règles préservent la légitimité de CapitalInvest à réclamer une partie des profits financiers au moment de la sortie sans avoir à s'impliquer sans limite.

«Le partage de la plus-value a un moment donné est toujours un moment délicat et il l'est d'autant moins qu'on a bien suivi la participation, [...] si on est resté un peu dans la passivité, la tentation est grande de modifier la part du gâteau. » (DP 8)

Nos résultats montrent que la légitimité des conseils de l'investisseur peut résulter non pas seulement d'un niveau d'engagement élevé (De Clercq et Sapienza 2006), mais aussi d'une adéquation avec les attentes des entrepreneurs qui demandent une attention forte de la part des DP uniquement sur des durées réduites.

\subsubsection{Rester modeste et s'adapter aux préférences du dirigeant}

Cultiver, chez les DP, un comportement empreint de modestie et d'adaptabilité à l'égard des entrepreneurs et de leurs préférences correspond à la troisième règle informelle qui gouverne le déploiement des relations entre les deux parties. Il est frappant de constater à quel point une attitude de déférence à l'égard des entrepreneurs est érigée en posture partagée par l'ensemble des investisseurs. Il s'agit avant tout, selon eux, de mettre en avant le dirigeant et de faire preuve d'empathie à son égard.

"Il faut savoir supporter les humeurs du chef d'entreprises [...]. Il faut être assez transparent en fait, et hermétique, et adaptable à toutes les situations. Eliminer toute forme d'affectivité et faire preuve d'une grande modestie. Ce n'est pas le plus facile car on a parfois tendance à vouloir se faire valoriser. » (DP 8)

Les DP prennent aussi grand soin de tenir compte et d'adapter leur comportement aux volontés, au style, à la personnalité, bref, aux préférences du dirigeant qu'ils ont en face d'eux.

"Ce qu'il peut y avoir entre l'investisseur et le chef d'entreprise est quand même lié au caractère du chef d'entreprise. Pas au nôtre car nous, notre métier, c'est de s'adapter à toutes les situations et à 
tous les comportements. [...] On est un peu des caméléons finalement.» (DP 6)

Cette modestie revendiquée par tous les DP est grandement encouragée par la division des tâches au sein de Capital-Invest, notamment la non-spécialisation sectorielle, et donc leur absence d'expertise opérationnelle. Au niveau collectif, l'agrégation de ces attitudes individuelles devient un facteur distinctif de Capital-Invest par rapport à ses concurrents parisiens ou internationaux, régulièrement perçus par les entrepreneurs comme hautains. Les DP évoquent souvent en entretien les reproches faits par les dirigeants de leur portefueille à de jeunes investisseurs de fonds nationaux, jugés trop sûrs d'eux et usant d'un vocabulaire financier déconnecté de la réalité du business. Le devoir de modestie se traduit y compris de manière surprenante au moment de la sortie par le fait que Capital-Invest a pour norme implicite la particularité de renoncer dans certains cas à chercher de manière offensive à maximiser ses gains immédiats et cela afin de ne pas froisser le dirigeant et gagner ainsi un soutien pour des collaborations futures.

"Si on fait un TRI de 19,5\% et pas de $20 \%$, on sera très content. On ne sera pas 'greedy' [...] C'est important de préserver une relation correcte à ce moment là [la sortie] et qu'il y ait un accord sur la valeur. Demain il peut y avoir une nouvelle opération. On peut de nouveau rentrer deux ans après être sorti. » (DP 6)

Ce type d'exemple en circulation au sein de Capital-Invest participe au fait de donner aux DP une image des dirigeants à traiter comme des alliées dont il faut s'attirer les bonnes grâces. Les normes de comportement ici décrites visent à construire une confiance avec l'entrepreneur en ne lui déplaisant jamais. Se mettre en position d'écoute et d'apprentissage, plutôt que chercher à démontrer une supériorité quelconque, permet en effet aux DP, à travers leurs interactions, de recueillir des informations de la part des dirigeants et donc de les contrôler mais de manière indirecte.

\section{Conclusion}

Cette recherche confirme qu'apporter autre chose que des fonds propres est effectivement vu par les capital investisseurs comme une condition sine qua non pour entretenir durablement une bonne relation 
avec l'entrepreneur et préserver des conditions optimales de sortie (Bygrave et Timmons 1992). Notre recherche complète les travaux qui soulignent l'importance des relations interpersonnelles entre entrepreneurs et capital investisseurs (De Clercq et Manigart 2007 ; De Clercq et Sapienza 2001, 2006), en mettant l'accent sur une dimension largement occultée : le rôle de l'organisation dans la construction de telles relations. L'article montre que le développement de liens interpersonnels n'est pas seulement le fruit d'affinités subjectives et purement individuelles entre deux personnalités en présence, mais qu'il résulte aussi de mécanismes de nature organisationnelle au niveau des sociétés de capital investissement. Ce développement correspond à un travail méthodique et systématique de la part des capital investisseurs tout au long des phases du cycle d'investissement, travail encadré et facilité par des règles formelles (e.g. réunions commerciales hebdomadaires obligatoires ; procédures collectives d'évaluation et de décision; dispositifs de partage des connaissances et des responsabilités; dispositifs de GRH mutualisant une partie des rémunérations). Le cas met ainsi en lumière l'encadrement du travail des DP par un ensemble de dispositifs organisationnels y compris plus informels comme des normes de comportement à l'image de la modestie qui sous-tendent la construction puis le développement d'une telle relation ( $c f$. Tableau 4 pour une synthèse).

Tableau 4-Mécanismes organisationnels de construction et de développement d'une relation interpersonnelle InvestisseursEntrepreneurs

\begin{tabular}{|c|c|c|}
\hline Etapes & $\begin{array}{l}\text { Règles, procédures et dispositifs encadrant le travail des DP } \\
\text { dans leurs relations avec les entrepreneurs }\end{array}$ & $\begin{array}{c}\text { Effets sur la } \\
\text { personnalisation des } \\
\text { relations }\end{array}$ \\
\hline \multirow{2}{*}{$\begin{array}{l}\text { Identification } \\
\text { \& Sélection }\end{array}$} & $\begin{array}{l}\text { Mission de prospection imposée aux DP engendrant des interactions } \\
\text { précoces et répétées avec des dirigeants cibles : } \\
-\quad \text { Obligation pour les jeunes recrues de développer un } \\
\text { - } \quad \text { nouveau réseau d'apporteurs d'affaires } \\
\text { - } \quad \text { Organision géographique du travail } \\
\end{array}$ & $\begin{array}{l}\text { - Capter une demande de } \\
\text { financement en } \\
\text { « séduisant » le dirigeant } \\
\text { d'entreprises cibles avant } \\
\text { la concurrence }\end{array}$ \\
\hline & $\begin{array}{l}\text { Institutionnalisation de l'octroi de conseils gracieux aux } \\
\text { entrepreneurs permise par: } \\
\text { - Pratiques de GRH : recrutement de DP «régionaux », } \\
\text { faible turn-over; dispositifs de rémunération } \\
\text { - } \quad \begin{array}{l}\text { Dispositifs de formalisation et de mutualisation des } \\
\text { connaissances sur les entreprises cibles }\end{array}\end{array}$ & $\begin{array}{l}\text { - Repérer les signaux de } \\
\text { coopération chez les } \\
\text { dirigeants }\end{array}$ \\
\hline
\end{tabular}




\begin{tabular}{|c|c|c|}
\hline $\begin{array}{l}\text { Evaluation \& } \\
\text { Structuration }\end{array}$ & $\begin{array}{l}\text { Préservation du caractère personnalisé de la relation malgré une } \\
\text { logique de dépersonnalisation à l'œuvre : } \\
\text { - Maintien du DP initial comme interlocuteur principal de } \\
\text { - } \quad \text { Sontrepreneur } \\
\text { - } \quad \text { Rapidité de la décision d'investissement } \\
\text { - } \quad \text { Procédure collective d'évaluation qui reste invisible pour } \\
\text { l'entrepreneur; } \\
\text { - } \quad \text { Offre de financement très personnalisée } \\
\text { - Sentiment de l'entrepreneur qu'il aura à ses côtés un décideur }\end{array}$ & $\begin{array}{l}\text { - Eviter les biais de } \\
\text { sélection grâce à la } \\
\text { dépersonnalisation de la } \\
\text { relation... } \\
\text { - ...sans toutefois remettre } \\
\text { en cause ouvertement le } \\
\text { caractère personnalisé de } \\
\text { cette relation }\end{array}$ \\
\hline $\begin{array}{l}\text { Activités Post- } \\
\text { investissement }\end{array}$ & 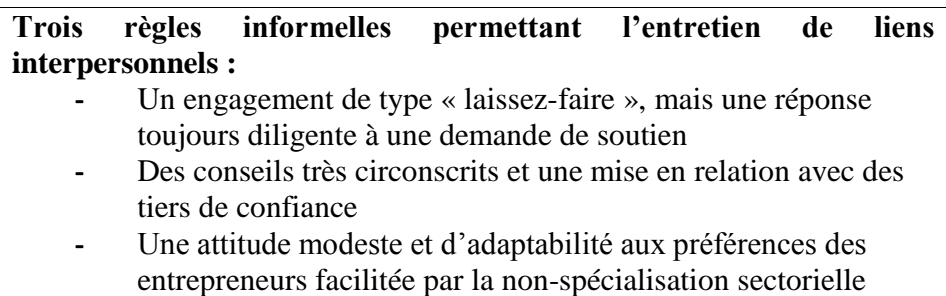 & $\begin{array}{l}\text { - Construire une légitimité } \\
\text { d'apporteur de conseils } \\
\text { - Exercice d'un contrôle de } \\
\text { proximité et non pas « ex } \\
\text { post» }\end{array}$ \\
\hline
\end{tabular}

Cet article montre comment une société de capital investissement peut poursuivre à la fois un objectif de personnalisation de ses relations avec les entrepreneurs tout en préservant la distance nécessaire à la prise de décisions objectives. Ce savoir-faire est particulièrement important dans la mesure où la personnalisation des relations investisseurs-entrepreneurs est gage de transmission de valeur ajoutée, tandis que la capacité à prendre du recul limite les risques de sélection adverse notamment en phase de pré-investissement. Il s'agit ainsi pour les sociétés de capital investissement de capter des demandes de financement en nombre relativement réduit, au moins pour les plus intéressantes, en «séduisant » des dirigeants sans se laisser entraîner par les liens parfois étroits tissés au cours du temps.

Cette étude complète également la littérature sur l'apport de valeur non financière (Large et Muegge 2008) par les capitaux investisseurs en étendant l'analyse aux phases de pré-investissement. Elle montre que l'établissement de relations interpersonnelles débute bien avant l'évaluation et la signature du contrat d'investissement. Elle souligne également l'interdépendance entre les phases du cycle d'investissement en matière de construction de relations interpersonnelles. Les interactions sociales qui permettent un transfert de valeur ajoutée non financière en phase de post-investissement reposent ainsi par exemple sur la légitimité du conseil des investisseurs qui s'établit dès la phase d'identification de l'entreprise. 
En terme d'intérêt pour les acteurs de la sphère économique, la caractérisation des modalités de construction d'une relation de qualité intéresse autant les capital investisseurs que les entrepreneurs: les premiers pour prendre en compte les effets déterminants de l'organisation du travail qu'ils se donnent sur les capacités individuelles de leurs DP à développer des relations de coopération avec les entrepreneurs ; les seconds pour être plus au fait des attendus des capital investisseurs au-delà des seuls critères financiers classiquement mis en avant. Ce cas illustre en outre les conditions d'un usage efficace de l'emploi de règles formelles et informelles afin d'orienter le comportement commercial d'une population de capital investisseurs autonomes. En effet, aucune des règles organisationnelles ne vise à prescrire de manière précise et étroite ce qui fait le succès d'une société de capital investissement régionale à savoir, le contact avec les dirigeants. En revanche, la manière d'approcher les dirigeants, le temps qu'il s'agit de leur consacrer avant et après la signature des deals, l'attitude propice à adopter lors des échanges et bien d'autres aspects à même d'orienter les comportements, sont finement encadrés par Capital-Invest et aident les DP à faire leur travail.

Reposant sur une étude de cas unique, les résultats présentés restent exploratoires. Ils invitent à approfondir l'analyse de la dimension sociale des échanges économiques dans le champ du capital investissement ainsi que des mécanismes engendrant la coopération entre les deux parties.

\section{Bibliographie}

Arthurs J.D. et Busenitz L.W. (2003), «The Boundaries and Limitations of Agency Theory and Stewardship Theory in the Venture Capitalist/Entrepreneur Relationship », Entrepreneurship, Theory \& Practice, vol. 28, n 2, p. 145-162.

Bonnet C. (2005), «La confiance entre capital-investisseurs et dirigeants:conséquences comportementales et influence sur la performance financière », Finance Contrôle Stratégie, vol. $8, \mathrm{n}^{\circ} 2$, p. 99-132.

Busenitz L.W., Fiet J.O. et Moesel D.D. (2004), « Reconsidering the Venture Capitalists' "Value Added" Proposition: An Interorganizational Learning Perspective », Journal of Business Venturing, vol. 19, $\mathrm{n}^{\circ} 6$, p. 787-807. 
Bygrave W.D. et Timmons J.A. (1992), Venture Capital at the Crossroads, Harvard Business School Press.

Crozier M. (1964), Le phénomène bureaucratique, Editions du Seuil.

Crozier M. et Friedberg E. (1977), L'acteur et le système: Les contraintes de l'action collective, Editions du Seuil.

De Clercq D. et Sapienza H.J. (2001), «The Creation of Relational Rents in Venture Capitalist-Entrepreneur Dyads », Venture Capital, vol. $3, \mathrm{n}^{\circ} 2$, p. 107-127.

De Clercq D. et Fried V.H. (2005), «Executive Forum: How Entrepreneurial Company Performance Can Be Improved through Venture Capitalists' Communication and Commitment», Venture Capital, vol. 7, n 3, p. 285-294.

De Clercq D. et Sapienza H.J. (2006), «Effects of Relational Capital and Commitment on Venture Capitalists' Perception of Portfolio Company Performance », Journal of Business Venturing, vol. 21, $\mathrm{n}^{\circ} 3$, p. 326-347.

De Clercq D. et Manigart S. (2007), «The Venture Capital Postinvestment Phase: Opening the Black Box of Involvement», in $\mathrm{H}$. Landström (ed.), Handbook of Research on Venture Capital, Edward Elgar.

Desbrières P. (2001), "La relation capital-investissement dans les firmes industrielles et commerciales », in G. Charreaux (ed.), Images de l'investissement. Au-delà de l'évaluation financière: une lecture organisationnelle et stratégique, Vuibert-FNEGE.

Desbrières P. et Broye G. (2000), "Critères d'évaluation des investisseurs en capital: le cas français », Finance Contrôle Stratégie, vol. 3, n 3, p. 5-43.

Eisenhardt K.M. et Graebner M.E. (2007), «Theory Building from Cases: Opportunities and Challenges », Academy of Management Journal, vol. 50, $\mathrm{n}^{\circ}$ 1, p. 25-32.

Ehrlich S.B., De Noble A.F., et Moore T. (1994), «After The Cash Arrives: A Comparative Study of Venture Capital and Private Investor Involvement in Entrepreneurial Firms », Journal of Business Venturing, vol. $9, \mathrm{n}^{\circ} 1$, p. 67-82.

Friedberg E. (1993), Le pouvoir et la règle, Editions du Seuil.

Fulghieri P. et Sevilir M. (2009), «Size and Focus of a Venture Capitalist's Portfolio », Review of Financial Studies, vol. 22, $\mathrm{n}^{\circ} 11$, p. 4643-4680.

Gifford S. (1997), «Limited Attention and the Role of the Venture Capitalist », Journal of Business Venturing, vol. 12, n 6, p. 459-482. 
Goffman E. (1973), La mise en scene de la vie quotidienne: La présentation de soi, Les Editions de Minuit.

Gorman M. et Sahlman W. A. (1989), "What Do Venture Capitalists Do? », Journal of Business Venturing, vol. 4, n 4, p. 231-248.

Gouldner, A.W. (1954), Patterns of Industrial Bureaucracy, Free Press.

Hellmann T. et Puri M. (2002), «Venture Capital and the Professionalization of Start-Up Firms: Empirical Evidence », Journal of Finance, vol. 57, n 1, p. 169-197.

Jackson III W.E., Bates T. et Bradford W.D. (2012), « Does Venture Capitalist Activism Improve Investment Performance? », Journal of Business Venturing, vol. 27, $\mathrm{n}^{\circ}$ 3, p. 342-354.

Kirzner I. (1973), Competition and Entrepreneurship, University of Chicago Press.

Knight F.H. (1921), Risk, Uncertainty, and Profit, Hart, Schaffner \& Marx, Houghton Mifflin Company.

Langley A. et Royer I. (2006), «Perspectives on Doing Case Study Research in Organizations », M@n@gement, vol. 9, n 3, p. 81-94.

Large D. et Muegge S. (2008), «Venture Capitalists' Non-financial Value-added: An Evaluation of the Evidence and Implications for Research », Venture Capital, vol. 10, n 1, p. 21-53.

Macmillan I.C., Kulow D.M. et Khoylian R. (1989), «Venture Capitalists' Involvement in their Investments: Extent and Performance », Journal of Business Venturing, vol. 4, $\mathrm{n}^{\circ}$ 1, p. 27-47.

Meyer J.W. et Rowan B. (1977), «Institutionalized Organizations: Formal Structure as Myth and Ceremony », American Journal of Sociology, vol. 83, $\mathrm{n}^{\circ}$ 2, p. 340-363.

Morgan R.M. et Hunt S.D. (1994), « The Commitment-Trust Theory of Relationship Marketing », Journal of Marketing, vol. 58, n 3, p. 20-38.

Merton R.K. (1940), «Bureaucratic Structure and Personality », Social Force, vol. 18, n $^{\circ} 4$, p. $560-568$.

Petty J.S. et Gruber M. (2011), " "In Pursuit of the Real Deal". A Longitudinal Study of VC Decision Making », Journal of Business Venturing, vol. 26, $\mathrm{n}^{\circ} 2$, p. 172-188.

Politis D. (2008), «Business Angels and Value-added: What Do we Know and Where Do we Go? », Venture Capital, vol. 10, n 2, p. $127-$ 147.

Rosenstein J. (1988), «The Board and Strategy: Venture Capital and High Technology », Journal of Business Venturing, vol. 3, $\mathrm{n}^{\circ} 2$, p. 159 170. 
Rosenstein J., Bruno Albert V., Bygrave William D. et Taylor, Natalie T. (1989), « Do Venture Capitalists on Boards of Portfolio Companies Add Value Besides Money? », Frontiers of Entrepreneurship Research, p. 216-229.

--- (1993), «The Ceo, Venture Capitalists, and the Board », Journal of Business Venturing, vol. 8, $\mathrm{n}^{\circ}$ 2, p. 99-113.

Sapienza H.J. (1992), «When Do Venture Capitalists Add Value?», Journal of Business Venturing, vol. 7, $\mathrm{n}^{\circ}$ 1, p. 9-27.

Sapienza H.J., Manigart S. et Vermeir W. (1996), «Venture Capitalist Governance and Value Added in Four Countries », Journal of Business Venturing, vol. 11, $n^{\circ}$ 6, p. 439-469.

Sapienza H.J. et Timmons J.A. (1989), «The Roles of Venture Capitalists in New Ventures: What Determines Their Importance?» Academy of Management Best Papers Proceedings.

Sapienza H.J. et Gupta (1994), « Impact of Agency Risks and Task Uncertainty on Venture Capitalist - CEO Interaction », Academy of Management Journal, vol. 37, n ${ }^{\circ}$, p. 1618-1632.

Selznick P. (1943), «An Approach to a Theory of Bureaucracy », American Sociological Review, vol. 8, $\mathrm{n}^{\circ}$ 1, p. 47-54.

Stévenot-Guéry A. (2007), "Capital-investissement en syndication: les enjeux en termes de gouvernance disciplinaire et cognitive à partir d'une étude de cas multi-sites », Finance Contrôle Stratégie, vol. 10, $\mathrm{n}^{\circ} 4$, p. 141-178.

Sweeting R.C. (1991), «U.K. Venture Capital Funds and the Funding of New Technology-Based Businesses: Process and Relationships », Journal of Management Studies, vol. 34, $\mathrm{n}^{\circ}$ 1, p. 125-152.

Tyebjee T. et Bruno A. (1984), «A Model of Venture Capitalist Investment Activity », Management Science, vol. 30, $\mathrm{n}^{\circ}$ 9, p. 10511066.

Weber M. (2003) Economie et Société. Les catégories de la sociologie. Pocket.

Yin R. K. (1994), Case Study Research: Design and Methods, Sage Publications. 


\section{Annexes}

\section{Guide d'entretien}

Contexte

- Quel est votre parcours professionnel ?

- Quelles sont vos responsabilités actuelles?

- Quels sont vos concurrents ?

- Retrouvez-vous vos concurrents dans les syndications ?

\section{Stratégie}

- Quelle stratégie commerciale avez-vous ?

- Est-ce que vous pourriez nous détailler d'une part comment a émergé cette stratégie ? et plus dans le détail, ce qu'elle recouvre?

- Quels mécanismes de levée de fond avez-vous?

- Il y a peu d'entreprises dans lesquelles vous avez une minorité de blocage : pourquoi ?

- Quelle est la durée typique de vos participations?

- Comment faites-vous pour suivre la diversité de votre portefeuille de participations?

- Est-ce qu'il y a des synergies opérationnelles entre les sociétés accompagnées ?

- Organisez-vous des rencontres entre les entreprises ?

Organisation du travail

- Comment est organisé le travail entre les directeurs de participations?

- Quels sont les critères de performance des DP ?

- Comment se passe un recrutement de DP ? Qu'attendez-vous d'un DP ?

\section{Sélection}

- Quels sont les critères de sélection des entreprises partenaires ?

- Quel est le taux de sélectivité ?

- Comment trouvez-vous les opportunités d'investissement ?

- Qui participe à la décision d'investir ou non ? 
Suivi

- Quels sont les critères de performance d'un investissement une fois celui-ci décidé ?

- Quelles sont les règles de suivi des participations ?

- Comment exercez-vous une influence auprès de vos participations?

- Sur quel domaine de la gestion intervenez-vous ?

- Quelles sont les expertises que vous revendiquez?

\section{Conclusion}

- Est-il envisageable de rencontrer un ou deux dirigeants de votre portefeuille?

- Est-il possible de venir assister à l'un de vos comités d'investissement?

\section{Processus organisationnel d'évaluation des entreprises chez Capital-Invest}

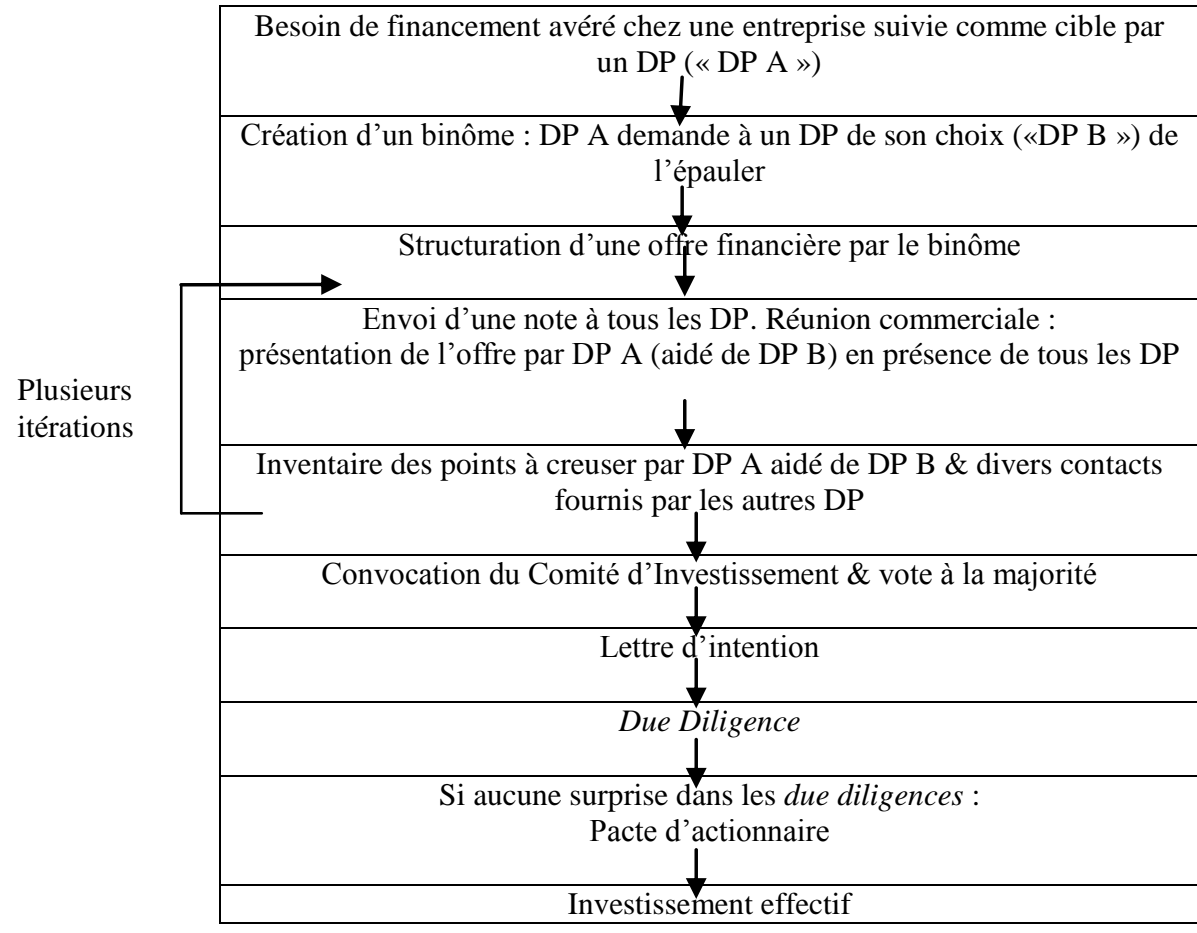

